\title{
Kinaesthetic and Cutaneous Contributions to the Perception of Compressibility
}

\author{
Wouter M. Bergmann Tiest and Astrid M.L. Kappers \\ Helmholtz Institute, Utrecht University, The Netherlands \\ W.M.BergmannTiest@phys .uu.nl
}

\begin{abstract}
Compressibility or hardness of objects is an important aspect in haptic perception. Both cutaneous and kinaesthetic information are used for the perception of compressibility. In this paper, the relative role of these contributions is investigated. This is done with psychophysical experiments using a purpose-made silicon rubber stimulus set. The fabrication and characterisation of the stimuli are described, as well as discrimination experiments with and without surface deformation of the stimuli. With the cutaneous cues of surface deformation present, the Weber fraction for hardness discrimination was 0.12 . When surface deformation was removed and only kinaesthetic cues were available, the Weber fraction doubled, suggesting that the cutaneous sense contributes almost three quarters to hardness perception, and the kinaesthetic just over one quarter, if the information is integrated in a statistically optimal fashion.
\end{abstract}

Keywords: Hardness, Softness, Compliance, Weber fraction.

\section{Introduction}

Haptic perception of the world around us consists of a number of aspects. We can for instance perceive shapes, textures and material properties of the objects that we encounter. One important material property is the compressibility or hardness of objects. We can find out information about objects by assessing this property, for example the ripeness of fruits or the air pressure in a bicycle tyre. For a realistic haptic experience, a good perception of compressibility is essential. There has been some research into the rendering of compressibility and some implementations in haptic devices, but a thorough understanding of this aspect is still lacking.

When interacting with a haptic display, this is usually done through some kind of rigid interface, e.g. a hand-held stylus or thimbles that slide onto he fingertips. This implies that the actual surface that is in direct contact with the skin does not deform when touched, even though the rendered, virtual surface might. Forces can be displayed to the user, but other cues associated with compressibility are generally not available. Possible other cues for the perception of compressibility include the shape and the size of the contact area and the dynamic deformation of the object surface, i.e. the way it forms itself around 
the finger. It is known that for the perception of softness and hardness, both tactile and kinaesthetic information are used [1], but the relative importance of these information sources has not been quantified. This quantification is very important regarding the design of haptic interfaces, but also with respect to more fundamental research in human perception. The kinaesthetic and cutaneous channels carry very different types of information; in the case of squeezing a compressible object, the kinaesthetic channel carries information about finger displacement and applied force, while information in the cutaneous channel has a more spatial character, comprising the size and force distribution of the contact area on the fingers. Analysis of the relative contribution of these channels can teach us about the way the information streams are integrated in the perceptual system.

The discrimination of compressibility, compliance or hardness has been investigated in a number of ways [2, 3, 4, 5, 6, 6], that are not all easily compared. One measure that can be used for comparing these studies is the Weber fraction, defined as the ratio between the discrimination threshold and the stimulus intensity. For instance, a Weber fraction of $\sim 0.1$ was reported by Tan et al. [6] using an apparatus consisting of two computer-controlled movable plates that the subject pressed toward each other in a pinch grasp. Somewhat worse performance, around 0.3, was reported by Freyberger and Färber 7] using a stimulus set of silicon rubber blocks that were squeezed between thumb and forefinger. It is surprising that the situation with Tan et al., in which only kinaesthetic information was available, yields a better performance than that with Freyberger and Färber. One would expect that the extra information available about the surface deformation of the silicon rubber would help the hardness discrimination, but this appears to be not the case at first sight. A possible explanation could be that the thresholds were not measured at the same reference hardness; in Tan et al. [6], the reference was $0.25 \mathrm{~N} / \mathrm{mm}$, while in Freyberger and Färber [7], references ranged from $1.2-56 \mathrm{~N} / \mathrm{mm}$. However, there was no indication that the latter thresholds would deviate from Weber's law at the soft end of the range. Another difference in the setups was that with Tan et al., the subject encountered a mechanical wall at a fixed position when squeezing the plates. Thus, subjects could have based their judgement on the terminal force or the total amount of mechanical work done. Indeed, when the authors minimised these cues, the thresholds went up to Weber fractions of 0.15-1.0. Still, it remains difficult to compare these two experiments with the purpose of extracting the contribution of surface deformation cues. In order to do that, it is desirable to perform an experiment with and without deformation of the contact surface while keeping the procedure and other circumstances the same.

In this paper, we report our investigations into the capacity of the human perceptual system to differentiate by means of touch between different magnitudes of compressibility. We have measured the discrimination threshold as a function of stimulus compressibility. Furthermore, the role of the cutaneous information has been measured by removing the contact surface deformation. 


\section{Stimuli}

For this experiment, a stimulus set was designed that encompassed a wide range in hardness and for which differences in other aspects, such as surface texture or shape, were minimised. The stimuli had to be easily grasped between thumb and forefinger. We decided to use silicon rubber cylinders of $4 \mathrm{~cm}$ length and $4 \mathrm{~cm}$ diameter (due to practical reasons the diameter of the final product was $41 \mathrm{~mm}$ ). In order to accurately measure discrimination thresholds, a finely spaced set of increasing hardness was necessary. Furthermore, since we wanted to know the threshold at several different reference hardnesses, different subsets were required, each consisting of one reference stimulus and eight test stimuli. Initially, three sets were fabricated, and a fourth set was added later.

\subsection{Fabrication}

Using a technique described in Freyberger and Färber [7], the different hardnesses were attained by mixing two types of pourable silicon rubber using different mixing ratios. We used condensation-curing, two-component silicon rubber that vulcanises at room temperature. The soft type was Elastosil M 4500 and the hard type was Elastosil M 4470 (Wacker Silicones). Catalysts (T 12 and T 40, respectively) were added to the uncured liquid rubber in a mass ratio of 3:100. Rubber and catalyst were mixed very well, and then the two types were mixed together in the desired ratio. For this ratio, the percentage of the hard type (M 4470) in the mixture was used as a parameter, ranging from 0-100\%. The required masses were determined with $0.01 \mathrm{~g}$ accuracy using a high-precision balance (Mettler P1200N). Taking into account the different densities of the two types of rubber and their mixing ratio, the required mass for each stimulus was calculated so as to keep the volume for all stimuli the same. The correct amount of mixture was then poured into a mold, which was made from a solid cylindrical plastic base (41 mm diameter) and flexible plastic foil, held together with sticky tape, see Fig. 11 After curing, the foil could be easily peeled off and disposed of, while the base could be re-used.

First, a small set of prototype stimuli was made to determine the required range of the sub-sets. This was done using magnitude estimation, yielding a relationship between the percentage of hard rubber and the perceived hardness of the stimulus. Using this relationship, mixing ratios were calculated for the test and reference stimuli in such a way, that the test stimuli close to the reference were perceptually spaced close together, while the test stimuli further away from the reference, at both ends of each sub-set, were perceptually spaced further apart. This ensured that the test stimuli were optimally spaced for the measurement of accurate psychometric curves. In this way, stimuli of 23 different hardnesses were created.

Later, when it became clear that stimuli were required that were even softer than the pure soft rubber stimulus (0\% hard rubber), a fourth set was made that consisted of a mixture of M 4500 and the silicone liquid AK 350 (Wacker Silicones), following Freyberger and Färber 7]. While the earlier set was designated with positive numbers (the percentage of hard rubber), the new set was 


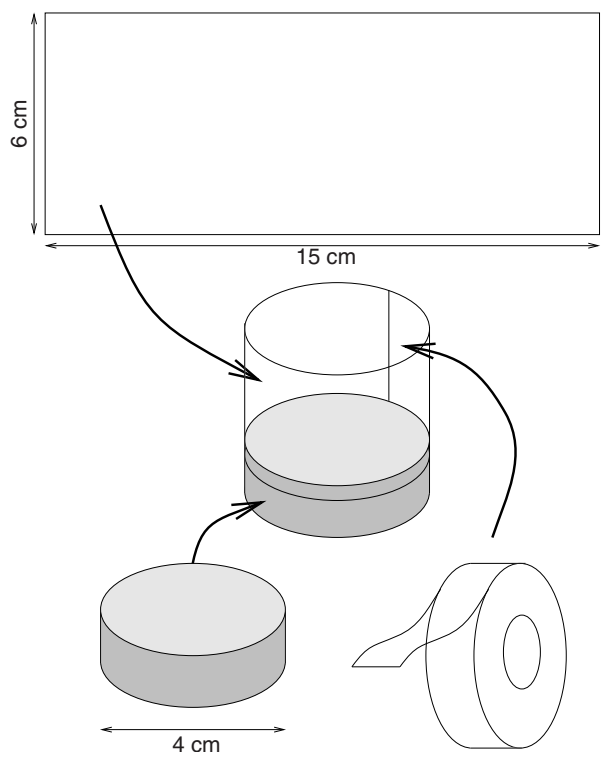

Fig. 1. Assembly drawing of the stimulus mold

designated with negative numbers, being the negative of the percentage of silicone liquid in the mixture. In this way, the numerical ordering corresponded to the hardness ordering.

\subsection{Characterisation}

In order to be able to express the thresholds in physical units, the stimuli had to be accurately characterised. For this, an Instron 5542 Universal Material Testing machine was used. This machine presses down on the stimuli while simultaneously measuring force and displacement. In this way, a force/displacement characteristic can be recorded. The measurements were performed in two ways: between two flat surfaces with the same area as the stimulus surface, and between two cylindrical brass probes with a cross section of $100 \mathrm{~mm}^{2}$. This surface area was chosen to be similar to the contact area of the thumb and forefinger contacting the stimulus in a pinch grasp.

In Fig. 2 (left), the force/displacement characteristics are shown for the measurement with the flat surfaces. The curves are fairly linear for forces greater than $20 \mathrm{~N}$. A linear fit was made to these curves above $20 \mathrm{~N}$ and their slopes are plotted in the right part of Fig. 2. Similar characteristics were measured with the probes. Because of the smaller surface area of the probes, less force is necessary for a similar displacement compared to the flat surfaces. Therefore, the slopes of those curves were fitted between 5 and $25 \mathrm{~N}$. The deviations from the monotonically rising trend that are visible in Fig. 2 (right) are probably due to inaccuracies in the measurements, since the mixing of the two types of rubber was done to 

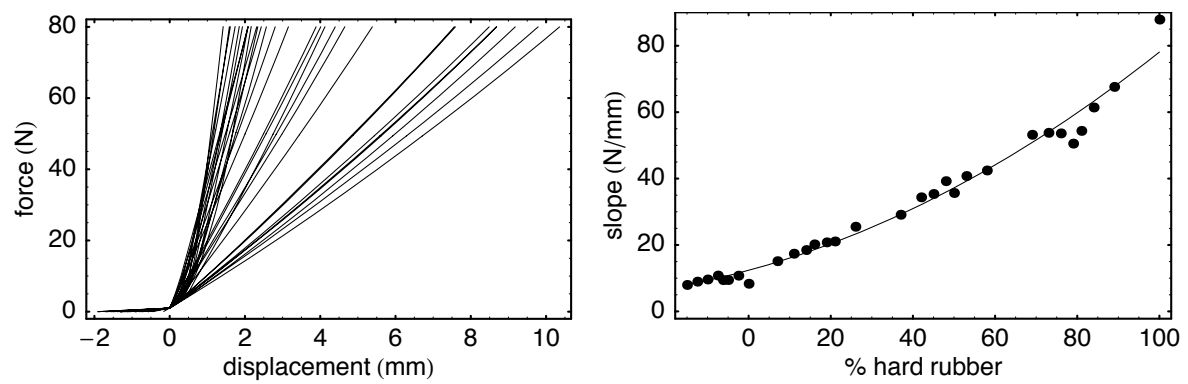

Fig. 2. Left: Force/displacement characteristics of the stimuli measured with flat surfaces. Right: Slopes of the curves on the left (dots) with a fitted function (solid line). The negative percentages refer to the mixtures of soft rubber and silicone liquid.

a very high standard. Indeed, the shape of the force/displacement characteristic was found to depend a little on the precise positioning of the probes. The accuracy of the stimulus set itself was confirmed by pilot experiments in which the stimuli had to be ordered according to increasing hardness. There were no systematic deviations from the intended ordering. In order to minimise the effect of these measurement inaccuracies, a quadratic function was fitted to the slopes in both sets of measurements. For the flat surfaces, the relationship between the percentage of hard rubber $h[\%]$ and the slope of the force/displacement characteristic $s[\mathrm{~N} / \mathrm{mm}]$ could be described by the equation

$$
s=0.0032 h^{2}+0.34 h+12 .
$$

For the brass probes, the equation

$$
s=0.0013 h^{2}+0.062 h+2.5
$$

holds. Using these equations, the thresholds found in the psychophysical experiments could be expressed in physical units. Also, by using the fitted functions instead of the individual measured hardnesses, the effect of random inaccuracies in the measured values was minimised.

\section{Psychophysical Experiments}

Two psychophysical experiments were performed with the stimuli described above. In the first, discrimination thresholds were measured at three reference hardnesses with direct contact between the fingers and the stimulus. In the second, the surface deformation cue was removed by inserting rigid metal discs between thumb and forefinger.

\subsection{Method}

Subjects. In both experiments, four different subjects participated. They were students at Utrecht University and were paid for their participation. There were 
three females and five males, and their mean age was $20.5 \pm 2.6$ years. All were strongly right-handed according to Coren's test [8].

Procedure. For each of the three hardness ranges, blindfolded subjects were repeatedly presented with the reference stimulus, the hardness of which was located in the centre of each range, and one of eight test stimuli. These were placed on their side on the table in front of the subject, one in front of the other. The locations of the test and reference stimuli were counterbalanced over trials, and the ordering of the trials was randomised. Subjects were asked to grab the stimuli between thumb and forefinger of their preferred hand. They could squeeze the stimuli and go back and forth as often as they liked. There was no time limit. The task was to indicate the harder of the two. Each combination of test and reference was tested 10 times, resulting in 240 trials per subject. The time taken was about 90-120 minutes, divided over two sessions. For the second experiment, $0.5 \mathrm{~mm}$ thick, $40 \mathrm{~mm}$ diameter stainless steel discs were stuck to both sides of the stimuli to eliminate any surface deformation. Since the force applied by the subject was now spread out over a much larger area, the stimuli felt much harder. In order to be able to compare the two experiments, the stimulus set was extended with an extra sub-set of stimuli at the soft end of the range. The hardest sub-set was not used in the second experiment.

Analysis. For each hardness range, the number of times that the test stimulus was found to be the hardest was plotted as a function of its hardness. In this way, a sigmoid trend is expected with the softest test stimuli never being perceived the hardest, the test stimuli very close to the reference about 5 out of 10 times, and the hardest test stimuli 10 out of 10 times. To this data, a cumulative Gaussian function was fitted of the form $f(x)=5 \operatorname{erf}(x / \sqrt{2} \sigma)+5$. The width $\sigma$ of this function corresponds to the $84 \%$ level and is a measure for the discrimination threshold.

\subsection{Results}

The results of the first experiment were plotted as a function of the stimulus hardness as measured using the brass probes. The data for the brass probes was used because they were measured in a way that is most compatible with the way the human subjects gripped the stimuli. That is, the deformation of the stimuli and the distribution of forces will be very similar between the mechanical measurements and pinching with the fingers. An example of an individual data plot is shown in Fig. 3 (left). By fitting the psychometric curve to the data, the value for the fit parameter $\sigma$ was determined. On the right hand side in Fig. 3 , the measured thresholds for all four subjects and the three hardness ranges in the first experiment are plotted. The average over subjects is indicated by the thick line. To this average, a linear fit was made with a slope of $0.12 \pm 0.01\left(R^{2}=0.99\right)$. Thus, in this range, the Weber fraction for hardness discrimination using a pinch grasp in the presence of surface deformation cues is about $12 \%$. 

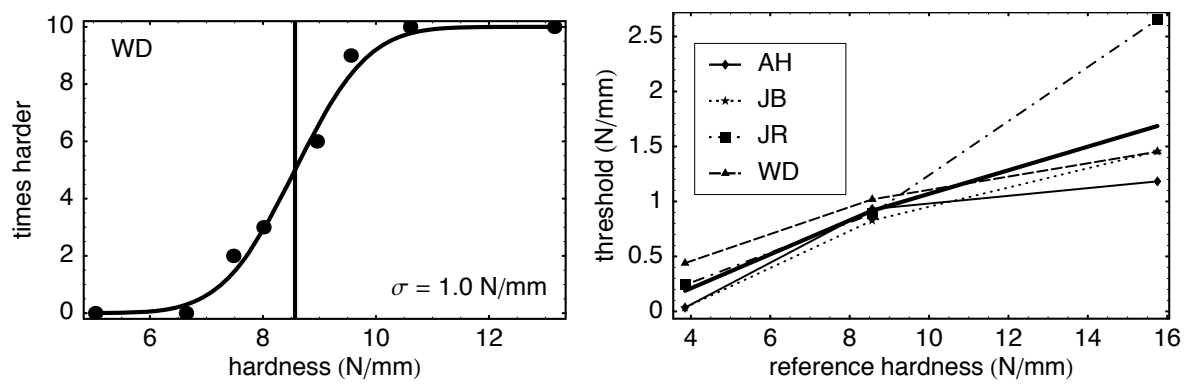

Fig. 3. Left: Example of a psychometric curve for the medium hardness range, for subject WD. The solid curve is a fitted function with the fitting parameter indicated in the bottom right corner. The vertical line indicates the position of the reference stimulus. Right: Hardness discrimination thresholds for four subjects and three reference levels. The thick line is the average over subjects.
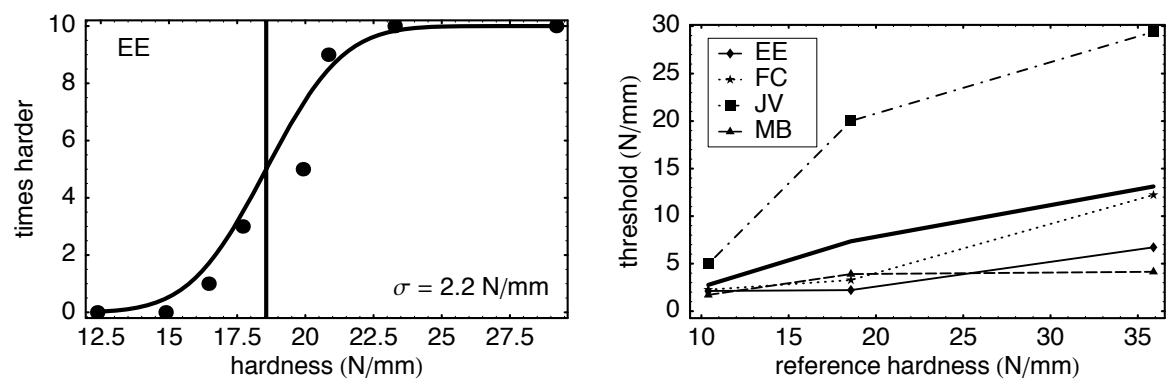

Fig. 4. Left: Example of a psychometric curve for the soft hardness range with the stainless steel discs present, for subject EE. The solid curve is a fitted function with the fitting parameter indicated in the bottom right corner. The vertical line indicates the position of the reference stimulus. Right: Hardness discrimination thresholds in the absence of surface deformation for four subjects and three reference levels. The thick line is the average over subjects.

For the second experiment, with the surface deformation removed, the psychophysical data were analysed in terms of the hardness measurements obtained using the flat surfaces. An example is shown in Fig. 4 (left). On the right, the thresholds for the four subjects in the second experiment are shown. The thick line indicates the average over the four subjects. Note the change in both the horizontal and the vertical scale compared to Fig. 3 (right). This is due to the fact that with the discs, the applied force is spread out over a much larger area than without the discs. Consequently, the force/displacement characteristic has a much steeper slope for the same stimulus with the discs than without. It is striking that subject JV has much higher thresholds than the other three subjects. The reason for this is unclear; during the experiment, subject JV's behaviour did not stand out from the others. However, in order to make a fair 


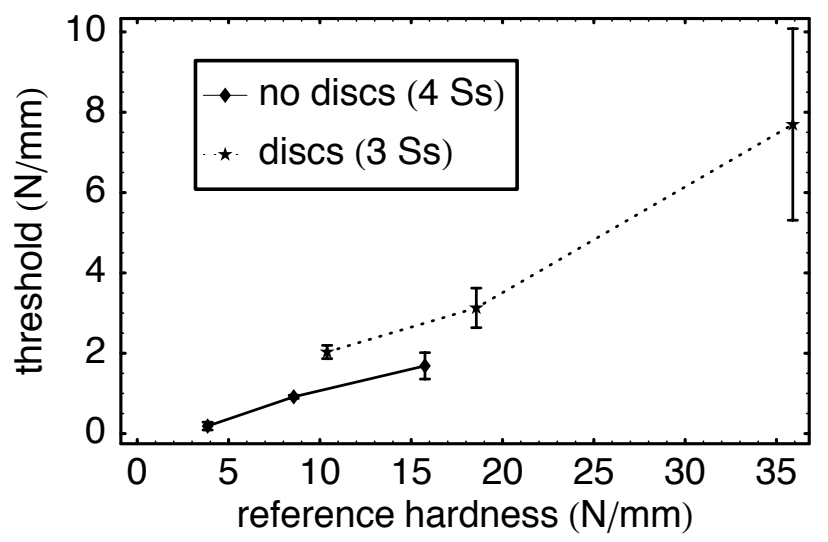

Fig. 5. Average thresholds for the first (diamonds) and second experiment (stars). The error bars indicate the standard error of the sample mean. For the second experiment, the data from subject JV are omitted.

comparison, it was decided to leave out subject JV's data from the remainder of the analysis. A linear fit to the average of the other three subjects yielded a slope of $0.23 \pm 0.03\left(R^{2}=0.98\right)$. Thus, without surface deformation, the Weber fraction for hardness discrimination is about $23 \%$.

In Fig. 5. the averages of each of the two experiments are plotted together. Due to the different hardness ranges, there is only a small overlap, but in that area, it appears that the removal of the surface deformation of the stimuli causes the thresholds to go up by a factor of two.

\section{Discussion and Conclusion}

In the first experiment, which comes closest to the 'natural' situation of squeezing an object, a Weber fraction of 0.12 was found. This is considerably lower than the value of $\sim 0.3$ reported by Freyberger and Färber 7]. The hardness ranges in that and the present experiment are quite similar, so that cannot be an explanation for the discrepancy. The fact that in Frevberger and Färber the stimuli were blocks compared to the cylinders of the present experiment also does not seem able to explain the difference. A possible reason for the better performance in the present experiment could be that subjects were not restricted by a time limit and could feel the test and reference stimuli as often as they liked. In this way, the values found here might be more indicative of the true limit of human performance in ideal circumstances, and should be interpreted as a lower limit.

When the surface deformation was removed, the Weber fraction went up to 0.23. This is identical to the value of 0.23 found by Jones and Hunter [5] and that of roughly 0.22 as found by Tan et al. [6] in their experiment with 'roving displacement', both of which used rigid interfaces. The value is almost twice as high as the one with surface deformation cues present. This confirms that 
for optimum hardness perception, both tactile and kinaesthetic information are necessary. This was already shown by Srinivasan and LaMotte [1], but the present experiments serve to accurately quantify this. Although the number of subjects is low, the high number of trials per subject and the agreement with earlier experiments ensure the reliability of these numbers.

The cutaneous and the kinaesthetic senses can be seen as two parallel information sources that are combined to produce the final perception, in this case of the hardness of an object. In the second experiment, with the discs, the cutaneous channel provided little useful information about the object hardness. We can therefore suppose that the Weber fraction of 0.23 found in that experiment is based solely on information from the kinaesthetic channel. If we know how the two channels are combined, we can calculate how much the two information sources contributed to the thresholds found in the first experiment. It has been shown that the combination of visual and haptic information is done in a statistically optimal fashion [9]. That is, using maximum-likelihood estimation, the variance of the combined information is minimised. This predicts that the threshold $\sigma_{A B}$ based on combined information from two information sources (A and $\mathrm{B}$ combined) is related to the thresholds based on single information sources $\left(\sigma_{A}\right.$ and $\left.\sigma_{B}\right)$ by

$$
\sigma_{A B}^{2}=\frac{\sigma_{A}^{2} \sigma_{B}^{2}}{\sigma_{A}^{2}+\sigma_{B}^{2}} .
$$

If we assume that this way of combining information, which Ernst and Banks [9] showed to hold for the visual and haptic senses, also holds for the cutaneous and kinaesthetic senses, we can calculate the predicted threshold for a hardness discrimination experiment in which only cutaneous cues and no kinaesthetic cues are available. This 'cutaneous threshold' $\sigma_{c}$ is given by

$$
\sigma_{c}=\frac{1}{\sqrt{\frac{1}{\sigma_{c k}^{2}}-\frac{1}{\sigma_{k}^{2}}}} \approx 0.14,
$$

where $\sigma_{k}=0.23$ and $\sigma_{c k}=0.12$ are the thresholds based on the kinaesthetic and combined senses, respectively. Thus, from the results of both experiments taken together, we predict that the Weber fraction for hardness discrimination based on cutaneous information alone is 0.14 , compared to the Weber fraction of 0.23 for kinaesthetic information alone. This implies that the weights used for combining the information (proportional to the reciprocal of the square of the threshold) are $73 \%$ cutaneous and $27 \%$ kinaesthetic. It might therefore be suggested that the cues of contact area change and force distribution, which are mediated by the cutaneous channel, make up almost three quarters of the information used to assess the hardness or compressibility of an object. Thus, when in a haptic display, a compressible surface is rendered using forces that are applied to the user by means of rigid actuators, the accuracy with which the surface is perceived, and therefore the realism of the display, must be severely impaired because no cutaneous information is available. A good assessment of the compressibility of a surface might be very important, e.g. in medical applications. 
Therefore, the development of a haptic display that speaks to the cutaneous sense as well as the kinaesthetic sense will be an important step forward and could potentially improve the accuracy of perception by a factor of two.

Acknowledgements. This work was supported by a grant from the Netherlands Organisation for Scientific Research (NWO). We thank dr. Niels Anten (Department of Biology) for the use of the Instron 5542.

\section{Reference}

1. Srinivasan, M.A., LaMotte, R.H.: Tactual discrimination of softness. Journal of Neurophysiology 73(1), 88-101 (1995)

2. Scott Blair, G.W., Coppen, F.M.V.: The subjective judgements of the elastic and plastic properties of soft bodies; the "differential thresholds" for viscosities and compression moduli. Proceedings of the Royal Society 128 B, 109-125 (1939)

3. Coppen, F.M.V.: The differential threshold for the subjective judgement of the elastic and plastic properties of soft bodies. British Journal of Psychology 32(3), 231-247 (1942)

4. Harper, R., Stevens, S.S.: Subjective hardness of compliant materials. Quarterly Journal of Experimental Psychology 16, 204-215 (1964)

5. Jones, L.A., Hunter, I.W.: A perceptual analysis of stiffness. Experimental Brain Research 79(1), 150-156 (1990)

6. Tan, H.Z., Durlach, N.I., Beauregard, G.L., Srinivasan, M.A.: Manual discrimination of compliance using active pinch grasp: the roles of force and work cues. Perception \& Psychophysics 57(4), 495-510 (1995)

7. Freyberger, F.K.B., Färber, B.: Compliance discrimination of deformable objects by squeezing with one and two fingers. In: Proc. EuroHaptics, pp. 271-276 (2006)

8. Coren, S.: The left-hander syndrome: the causes and consequences of lefthandedness. Vintage Books, New York (1993)

9. Ernst, M.O., Banks, M.S.: Humans integrate visual and haptic information in a statistically optimal fashion. Nature 415(6870), 429-433 (2002) 